\title{
Impacto psicosocial de la diabetes mellitus tipo 1 en niños, adolescentes y sus familias. Revisión de la literatura
}

\author{
Psychosocial impact of type 1 diabetes mellitus in children, adolescents \\ and their families. Literature review
}

Rocío Henríquez-Tejo ${ }^{a}$, Ricardo Cartes-Velásquez ${ }^{\mathrm{b}, \mathrm{c}}$

${ }^{a}$ Cirujano Dentista, Fundación Kimntrum, Chile

${ }^{\text {bC } C i r u j a n o ~ D e n t i s t a, ~ F a c u l t a d ~ d e ~ O d o n t o l o g i ́ a, ~ U n i v e r s i d a d ~ A n d r e s ~ B e l l o, ~ C o n c e p c i o ́ n, ~ C h i l e ~}$

‘Psicólogo, Magíster en Salud Pública, Universidad Autónoma de Chile, Temuco, Chile

Recibido el 03 de octubre de 2017, aceptado el 23 de febrero de 2018

\section{Resumen}

La diabetes mellitus tipo 1 es la patología endocrina crónica más común en niños. El tratamiento incluye dieta, actividad física, medicación con insulina y un autocontrol adecuado. Este autocontrol puede ser dificultoso, provocando que niños, adolescentes y sus familias sufran diversas complicaciones psicosociales. Existe una relación inversa entre autocontrol y presencia de complicaciones psicosociales, siendo los principales problemas ansiedad y depresión, donde los adolescentes llegan a ser 2,3 veces más propensos a presentar problemas de salud mental. Las familias se ven afectadas inicialmente en el período de debut por un estado de shock, con sentimientos de angustia e ira. Los necesarios cambios de hábitos y estilos de vida pueden generar problemas psicosociales entre los que destacan trastornos ansiosos, depresivos y alimenticios. Posteriormente, el niño o adolescente y su grupo familiar pueden transitar a un nuevo equilibrio caracterizado por un buen autocontrol y adherencia al tratamiento o profundizar los trastornos individuales y grupales, trastornos que pueden reaparecer, especialmente en la adolescencia. El tratamiento integral de la diabetes mellitus tipo 1 requiere atender estos aspectos mediante equipos multidisciplinarios que incluyen profesionales médicos y del ámbito psicosocial. En esta revisión se analizan los principales aspectos relacionados al impacto psicosocial en niños y adolescentes con diabetes mellitus tipo 1 y sus familias.

\footnotetext{
Abstract

Type 1 diabetes mellitus is the most common chronic endocrine pathology among children. Treatment includes diet, physical activity, insulin medication, and proper self-control. This self-control may be difficult, resulting in children, adolescents and their families suffering diverse psychosocial complications. There is an inverse relationship between self-control and psychosocial complications, the main problems being anxiety and depression, where adolescents are 2.3 times more likely to have mental
}

Palabras clave: Diabetes mellitus tipo 1; impacto psicosocial; familia; adolescencia

\section{Keywords:}

Diabetes mellitus type 1; psychosocial impact; family 
health problems. Families are initially affected, in the debut period by a state of shock, with feelings of distress and anger. The necesary changes in habits and lifestyles can lead to psychosocial problems, including anxiety, depression and eating disorders. Subsequently, the child or adolescent and his or her family group may move into new balance characterized by good self-control and adherence to tratment, or deepen individual and group disorders which may reappear, especially in adolescence. The comprehensive treatment of type 1 diabetes mellitus requires addressing these aspects through multidisciplinary teams which include medical and phychosocial professionals. This review analyses the main aspects related to the psychosocial impact of diabetes mellitus type 1 among children, adolescents and their families.

\section{Introducción}

La diabetes mellitus tipo 1 (DMT1) es una de las patologías crónicas más comunes que afectan a los niños, siendo compleja e invasiva ${ }^{1,2}$. Su tratamiento debe contemplar una dieta alimenticia, regulación diaria de la actividad física, el uso adecuado de insulinoterapia y el control glicémico ${ }^{3}$. Un control inadecuado puede afectar el desarrollo, aumentar las comorbilidades, disminuir la esperanza de vida y aumentar el riesgo de complicaciones agudas y crónicas relacionadas con la diabetes $^{4}$. La responsabilidad por el cuidado de los niños con diabetes implica un impacto de dimensiones psicosociales, tanto en el niño como en su familia ${ }^{5}$.

Esta enfermedad, al ser crónica, afecta las distintas etapas de la vida de las personas que la padecen. La adolescencia es considerada una de las etapas más complicadas, debido a todos los cambios que se sufren, tanto fisiológicos como psicosociales, estando los jóvenes con diabetes propensos a mayores tasas de estrés y enfermedades psicológicas ${ }^{6,7}$. Dado lo anterior, se recomienda realizar un acompañamiento psicológico, especialmente en esta etapa, adoptando un enfoque más biopsicosocial y ambiental. De esta forma se lograrán mejores resultados en el control de la enfermedad y en la calidad de vida ${ }^{5}$.

El objetivo de este artículo es presentar una revisión del impacto psicosocial de la diabetes mellitus tipo 1 en niños, adolescentes y sus familias.

\section{Aspectos clínicos y epidemiológicos}

La DMT1 deriva de la destrucción autoinmune de las células beta del páncreas, lo que lleva a una deficiencia completa de insulina, por lo cual las personas deben auto administrarse insulina exógena. Se presenta comúnmente en la infancia, aunque algunas personas son diagnosticadas siendo adultos. La DMT1 ocurre en individuos genéticamente susceptibles, siendo activada por agentes ambientales, los cuales son desconocidos. Se cree que el proceso autoinmune ocurre durante meses o años antes de que se produzcan síntomas clínicos (poliuria, polidipsia, polifagia, pérdida de peso) y se diagnostique hiperglicemia ${ }^{1}$. Es una enfermedad compleja e invasiva de la infancia ${ }^{2}$, de difícil control pues, para el individuo afectado, conlleva variadas complicaciones tanto físicas, clínicas y psicosociales 4 .

La Asociación Americana de la Diabetes (ADA) y la Organización Mundial de la Salud (OMS) tiene como criterios diagnósticos: glicemia en ayunas con un nivel de $126 \mathrm{mg} / \mathrm{dL}$ por lo menos dos veces, glicemia a las $2 \mathrm{~h}$ post $75 \mathrm{~g}$ de glucosa mayor a $200 \mathrm{mg} / \mathrm{dL}$, síntomas de diabetes y glicemia mayor a $200 \mathrm{mg} / \mathrm{dL}$, o hemoglobina glicosilada $(\mathrm{HbAlc})>6,5 \%{ }^{1}$.

La incidencia de DMT1 está aumentando en todo el mundo ${ }^{1,8,9}$, siendo esta enfermedad la tercera condición crónica más común en la infancia ${ }^{2}$. Es por esto por lo que el año 2006 la ONU puso como foco a la diabetes y la denominó como una de las principales amenazas para la salud mundial ${ }^{5}$. Se plantea que este aumento es un reflejo del estilo de vida moderno?.

El autocontrol de la DMT1 es primordial para disminuir los riesgos y complicaciones a corto y largo plazo. Para llevar un buen autocontrol debe haber un equilibrio entre la planificación en dieta alimenticia, regulación diaria de la actividad física, el uso adecuado de insulinoterapia y el control glicémico óptimo, pero este autocontrol es difícil de lograr ${ }^{2,4}$.

Actualmente, el objetivo principal es mantener un buen control glicémico y nivel de calidad de vida ${ }^{7}$. Para esto, los pacientes deben inyectarse varias veces en el día y recibir insulina basal o administrar insulina subcutánea continua durante el día, mediante bomba infusora ${ }^{3}$.

Cuando los niños comienzan con esta enfermedad, la mayoría requiere hospitalización con terapia intravenosa para tratar la cetoacidosis diabética y los problemas de deshidratación. Además de compensar al paciente, se debe comenzar con la insulinoterapia, para esto se imparte educación integral de los aspectos prácticos y teóricos del autocontrol que deben llevar ${ }^{10}$.

El examen de elección para ver el cuidado que ha tenido el paciente es la $\mathrm{HbAcl}$, el objetivo para pacientes diabéticos es de $58 \mathrm{mmol} / \mathrm{mol}(7,5 \%)$ o menos ${ }^{2}$. Gracias a los nuevos avances en tipos de insulina y for- 
mas de administración de ésta, la HbAlc media y el porcentaje de niños y adolescentes que alcanzaron el objetivo del 7,5\% de HbA1c ha avanzado un poco ${ }^{11}$. Valores altos de HbA1c están asociados con problemas cardiovasculares, neuropatías, nefropatía, retinopatía, problemas periodontales, disfunción eréctil, entre otras $^{1,2}$.

La adhesión a los controles de glucosa, la administración de insulina y la cuidadosa evaluación dietética es esencial, por lo que estos pacientes son periódicamente evaluados por médicos, enfermeras y nutricionista $^{1}$. A diferencia de otras patologías, donde las decisiones sobre el cuidado son tomadas exclusivamente por los especialistas médicos, muchas de las responsabilidades para el autocuidado diario de la DMT1 están a cargo de los jóvenes y sus familias, incluso inmediatamente después del diagnóstico ${ }^{5}$. En estos pacientes existe un bajo bienestar familiar, un alto impacto en la vida social, así como la presencia de conflictos familiares ${ }^{12}$; elementos que muchas veces no son evaluados por los profesionales. Un estudio alemán muestra que, a pesar de las recomendaciones de proporcionar una evaluación de salud mental y un tratamiento adecuado, solo el $28 \%$ de los jóvenes con sintomatología depresiva elevada informaron haber recibido atención psicológica $^{13}$.

Los resultados positivos para la salud vienen de una efectiva comunicación entre los profesionales y los padres, pero esta comunicación en la práctica no es ideal $^{14}$. Cuando las habilidades de comunicación de los profesionales son deficientes, pueden llevar a los padres a dejar de asistir a controles. Es importante que el equipo de salud se capacite para atender las necesidades emocionales de niños, adolescentes y cuidadores, mejorando su adherencia a los tratamientos ${ }^{2}$.

\section{Costos personales y sociales}

La DMT1 tiene un elevado costo para las personas y los presupuestos nacionales de salud, los cuales van aumentando con el aumento de su incidencia y la falta de adherencia a los tratamientos ${ }^{15}$.

Los costos directos incluyen a los medicamentos, servicios de consulta y diagnóstico y, en menor grado, costo de hospitalización por descompensación. También está el costo debido a las principales complicaciones, siendo el mayor relacionado a nefropatía diabética, siguiéndole retinopatía, enfermedad cardiovascular, neuropatía diabética y finalmente enfermedad vascular periférica. Los costos indirectos tienen relación con la mortalidad prematura, discapacidad permanente y discapacidad temporal. En México, el mayor impacto está en el bolsillo de los usuarios con un 51\% de los gastos totales ${ }^{16}$. En Grecia, el factor de mayor costo di- recto era el uso de la bomba de insulina, pero esto a su vez se asociaba con un mejor control metabólico, lo que reducía los costos a largo plazo ${ }^{17}$.

La evidencia indica la necesidad de diseñar mecanismos para controlar y reducir los costos asociados a la DMT1, particularmente los costos de sus complicaciones ${ }^{16}$. Los costos se deben ver como una inversión de capital de salud, con un mayor gasto en prevención primaria (específicamente en la diabetes mellitus tipo 2 ) y en la prevención secundaria de complicaciones ${ }^{18}$.

\section{Tratamiento actual de la DMT1 en Chile}

Desde el 2005, año en el que comienza a regir el régimen de garantías en salud (AUGE), la DMT1 tiene atención sanitaria garantizada en Chile. El diagnóstico, tratamiento, complicaciones, educación y aspectos nutricionales son garantizados por ley, con los principios que rigen el AUGE: acceso, oportunidad, protección financiera y calidad ${ }^{19}$.

La Guía Clínica GES explicita: "El cuidado por un equipo multidisciplinario es la práctica clínica establecida para las personas de todas las edades con diabetes tipo 1. El equipo incluye: la persona con diabetes, su familia o cuidador; médico especialista, diabetólogo o endocrinólogo pediátrico o de adulto, alternativamente un médico pediatra o internista capacitado en el manejo de niños, adolescentes o adultos con diabetes, según corresponda; educador en diabetes (en Chile, esta función habitualmente la cumple una enfermera capacitada); nutricionista capacitada en diabetes; psicólogo; asistente social"'19.

Aquí además se nombra que los integrantes del equipo deben cumplir labores de educación, nutrición, tratamiento, identificación y manejo de las complicaciones, consejería y apoyo psicológico ${ }^{19}$.

El problema es que en el Listado Especifico de Prestaciones (LEP) GES en el tratamiento solo incluye "consulta de especialidades en Medicina Interna y Subespecialidades, Oftalmología, Neurología, Oncología, consulta o control por enfermera matrona o nutricionista". No se incluye profesionales psicosociales, como psicólogo o asistente social ${ }^{20}$.

Recientemente se ha avanzado en el tratamiento de la DMT1, la Ley para el Otorgamiento de las Prestaciones que cuentan con el Sistema de Protección Financiera para Diagnósticos y Tratamientos de Alto Costo (Ley 20.850, mejor conocida como "Ricarte Soto") incluyó el tratamiento basado en la administración de insulina, a través de infusores subcutáneos continuos de insulina (bombas de insulina) para personas con diagnóstico de DM1 inestable severa. En esta prestación se entrega bomba de insulina junto a todos sus insumos, pero el seguimiento no se encuentra garantizado, por 
lo cual no existe una evaluación del impacto y aspectos psicosociales que conlleva este cambio de tratamiento $^{21}$.

\section{Repercusiones psicosociales}

Para muchas familias el debut o diagnóstico de DMT1 genera un gran impacto, provocando incluso a un trauma significativo. Esto resulta en un estado de shock con sentimientos de dolor, ira y aislamiento debido a la naturaleza compleja, implacable e invasiva de la enfermedad y el enfrentamiento a una reconstrucción de una nueva vida normal ${ }^{1,2,5,22}$.

Además de los cambios en los hábitos familiares y estilos de vida, la incertidumbre sobre el futuro de su hijo, los mitos y pensamientos sobre complicaciones agudas y crónicas, hacen que se generen diversos problemas psicosociales para el niño o joven diagnosticado, y su ambiente cercano ${ }^{4}$. El nivel de apoyo ofrecido a los padres en el momento del diagnóstico es fundamental para su capacidad de afrontamiento a largo plazo. Si se dispone de un apoyo psicosocial adecuado, se pueden construir conocimientos y confianza, lo que conduce a una mayor adherencia al tratamiento, un mejor control de la glicemia, una mejor percepción general de la calidad de vida y una disminución de las complicaciones 5 .

Durante el período pediátrico, los padres asumen la responsabilidad de los controles de glicemia, la administración de insulina y la planificación de comidas, convirtiéndose también en pacientes. Esto provoca demandas muy importantes a los miembros de la familia, enfrentando diferentes desafíos en cada etapa de crecimiento y desarrollo ${ }^{2,5}$. Estas responsabilidades parentales pueden llevar a estrés, generando síntomas de burnout, principalmente en las madres. Los padres y madres llevan esa carga de criar un niño con diabetes, experimentando muchas veces culpa y preocupación por las hipoglicemias y complicaciones a futuro ${ }^{23,24}$.

Algunas familias pueden gestionar de buena forma las nuevas responsabilidades de tener un integrante con DMT1. Sin embargo, para otras familias se transforma en una carga terrible si además se enfrentan a otros problemas como pobreza, desempleo, falta de tiempo o tener otros miembros con enfermedades crónicas 5 . El impacto en la vida familiar se reconoce con un factor que afecta el cuidado de la DMT1, ya sea interfiriendo en la capacidad de monitoreo de los padres o creando un ambiente hostil ${ }^{2,25}$. Incluso las familias con niños de muy poca edad pueden llegar al aislamiento social debido al miedo, sobre todo al de hipoglicemia. Por estos motivos es de gran importancia el apoyo psicosocial apropiado para la familia y comunidad en el que el niño se desenvuelve ${ }^{5,26}$.
Muchas veces no solo afecta a los padres o tutores del niño que padece DMT1, sino que también hay problemas de comportamientos, baja autoestima y trastornos emocionales en los hermanos, aunque algunos pueden aumentar su madurez y ganar fuerza con la situación ${ }^{27}$.

A medida que los niños van creciendo, esta responsabilidad de llevar un buen control es llevada en parte por los compañeros y profesores en su escuela, quienes desempeñan papeles importantes ${ }^{2,5}$. Este traspaso de responsabilidades puede provocar un grado de ansiedad tal, que los padres pueden generar síntomas de burnout ${ }^{28,29}$.

\section{Adolescencia y problemas psicosociales}

Durante la adolescencia, los jóvenes con DMT1 a menudo buscan la independencia y en ocasiones se rebelan contra los comportamientos de autocuidado. Es aquí donde comienzan las complicaciones, produciéndose un deterioro significativo en la adherencia al tratamiento, así como el control glicémico en adultos jóvenes con DMT1 $1^{1,2}$.

La adolescencia es la fase más difícil en la vida para tratar la diabetes debido a procesos tanto fisiológicos como psicosociales ${ }^{6.7}$. Para un joven sin alguna patología crónica, significa de por sí cambios en el estado de ánimo, indiferencia y conductas impulsivas ${ }^{5}$. El control glicémico empeora, entre otras razones, debido a la resistencia a la insulina fisiológica normal que es causada por los altos niveles de hormona del crecimiento ${ }^{30}$. Esto hace que para un adolescente con DMT1 el tratamiento diario sea aún más difícil de controlar ${ }^{31}$.

Existe una mayor incidencia de problemas psicosociales en comparación a la población general, que alcanza a 2,3 veces ${ }^{32}$. No es raro que muchos adolescentes, presentan sentimientos de ira, miedo, infelicidad o incluso crisis de angustia por la presencia de la $\mathrm{DMT}^{2}{ }^{2}$. Esta angustia emocional lleva a un rechazo de la enfermedad, que desarrolla problemas con las familias y el entorno más cercano. Muchos de los pacientes con diabetes relatan que tienen preocupación por las reacciones y los problemas con la familia, amigos y compañeros que no son diabéticos ${ }^{1}$.

En los jóvenes diabéticos se ha reportado que los trastornos psiquiátricos más frecuentes son la ansiedad, depresión y trastornos alimenticios ${ }^{1,5,32}$. En Chile, jóvenes con DMT1 presentaron altos niveles de estrés, debido a los serios y complejos desafíos psicológicos y conductas, los que pueden llevar a presentar síntomas depresivos, mala adherencia a la insulinoterapia y llegar así a un pobre control metabólico ${ }^{31}$. Las principales fuentes de estrés son el estrés emocional y el estrés asociado al tratamiento. El estrés psicológico puede ejercer sus efectos de modo directo o indirecto, 
ya sea interfiriendo la ejecución de las conductas pilares del tratamiento o bien por medio de la activación persistente del eje adrenérgico hipotalámico pituitario, generando una cascada de eventos fisiológicos que finalmente conllevan la liberación de glucocorticoides y, por tanto, elevación de niveles de glicemia ${ }^{33}$. Los jóvenes con DMT1 tienen un 33\% mayor riesgo de presentar depresión ${ }^{5}$, que se asocia con un mayor riesgo de complicaciones crónicas ${ }^{31}$.

Ahora bien, la adolescencia tardía puede ser un momento especialmente difícil para las personas con DMT1 producto de las transiciones normativas en la vida escolar y familiar ${ }^{31}$. La presencia de conflictos familiares lleva a una mala adherencia al tratamiento y peor autocontrol, todo ello asociado con un bajo nivel educacional familiar y un bajo nivel de participación familiar en la atención del joven ${ }^{5}$.

Muchos adolescentes dejan de lado la insulinoterapia (especialmente las mujeres) para perder peso; la no inyección de insulina hace que empiecen a ocupar las reservas de grasa de su cuerpo, lo que produce un adelgazamiento y un alza peligrosa de glicemia ${ }^{5}$.

Otro tema de cuidado es el consumo de alcohol, existiendo una asociación significativa entre este y un peor control glicémico. Además de esta complicación, existe mayor riesgo de situaciones de hipoglicemia y cetoacidosis posterior al consumo de alcohol. Por otra parte, la alteración de la consciencia con el consumo excesivo de alcohol lleva a que terceros confundan la hipoglicemia con intoxicación por alcohol ${ }^{34}$.

El cambio de atención pediátrica a adulta dificulta mantener un control glicémico y el seguimiento médi$\mathrm{Co}^{35}$. Esta transición presenta muchos retos, por lo que muchos adolescentes y adultos jóvenes con DMT1 se sienten agobiados y desanimados por el régimen que llevan. La responsabilidad ahora recae en ellos y no en sus padres, asociándose esta transición con un mayor riesgo de eventos adversos ${ }^{32,35}$. Lo anterior, lleva a una disminución en la motivación, empeorando el autocontrol, los controles glicémicos ${ }^{1,2,30,32,36}$ y la calidad de vida $^{7}$. Paradójicamente, en algunos casos esta transición puede promover la independencia y responsabilidad del adolescente ${ }^{37}$.

Así es como la Sociedad Chilena de Pediatría generó recomendaciones para la transición de la atención de servicios pediátricos a servicios de adultos en pacientes con enfermedades crónicas. En ellas, se destaca que hay una falta de diseño e implementación de políticas que ayuden a este problema, donde es importante constituir un equipo multidisciplinario que coordine los controles, fomente las redes de apoyo y genere actividades de continuidad de la atención. También se señala que es necesario generar recursos de apoyo emocional que incluyan consejería, psicoeducación, apoyo psicológico, entre otros ${ }^{38}$.
Si bien los pacientes con DMT1 sufren distintos sentimientos de rechazo y complicaciones psicosociales con su enfermedad, estos tienen percepciones de mayor autocontrol, mayor confianza y comprensión de su enfermedad y mejor capacidad de administrar su tratamiento respecto a los pacientes con DMT2 4 .

De todas maneras, es importante la evaluación de forma regular de los aspectos biopsicosociales y calidad de vida, tanto del adolescente como de su núcleo familiar, para así evitar la depresión, ansiedad o problemas alimenticios, para no caer en un mal autocontrol que pueda llevar a complicaciones crónicas, que en el largo plazo reducen la calidad de vida ${ }^{5}$. Es así como según los "Estándares de Atención Médica en la Diabetes 2016" de la Asociación Americana de Diabetes (ADA) ${ }^{39}$ (Standards of Medical Care in Diabetes-2016 Abridged for Primary Care Providers) se recomienda que exista una detección sistemática de problemas psicosociales, en la cual se incluyen depresión, ansiedad, angustia y trastornos alimentarios ${ }^{1}$.

Es de suma importancia realizar intervenciones tempranas de este conjunto de trastornos psicológicos, debido a que se ha relacionado con mejores resultados tanto psiquiátricos como metabólicos, además ayuda a reducir las complicaciones agudas de la DMT1 ${ }^{32}$. Otro aspecto fundamental es realizar la toma de decisiones en relación al tratamiento, junto con el adolescente, lo cual ayudaría a llevar un mejor autocontrol, generando mayores habilidades en el joven ${ }^{6}$.

\section{Atención psicosocial}

El tratamiento de la DMT1 debe incluir apoyo psicosocial como tratamiento ${ }^{5}$. El tratamiento integral debe incluir los problemas psicosociales para lograr un bienestar y de esta manera prevenir complicaciones futuras $^{40}$. El equipo médico debe contar con especialistas en salud mental, para así generar programas realmente integrales, que tengan un impacto en los individuos ${ }^{40}$. Muchos médicos consideran que el sistema carece de servicios de salud mental que apoyen a los pacientes diabéticos, y que estos necesitan apoyo psicosocial para construir una vida normal y un ambiente saludable en su hogar, para tener un desarrollo físico ideal, madurez emocional y cognitiva ${ }^{5}$.

La respuesta de los servicios y profesionales en relación con temas psicológicos tiene un efecto en el fortalecimiento de la adhesión al tratamiento y autocontrol $^{41}$. En este contexto, es imprescindible destacar la importancia de la comunicación y el apoyo emocional al paciente y su familia ${ }^{2,32}$, ya que el médico o profesional que trata al niño y al adolescente con DMT1 tiene un papel fundamental en pesquisar la necesidad de asistencia psicológica ${ }^{40}$. Los profesionales deben consi- 
derar los aspectos sociales que rodean al niño y adolescente $^{41}$, incluir el uso de instrumentos de detección que evalúen relación de autocontrol con funcionamiento cognitivo y problemas psicológicos ${ }^{42}$. La medicina debe velar por el cuidado de la persona entera, no solo de los aspectos físicos ${ }^{43}$.

Este apoyo y tratamiento psicosocial no solo debe ser duradero, sino apropiado para la edad sobre todo en la transición de la infancia a la adolescencia y luego a la etapa de adulto joven, debido a que se da lugar a menos ansiedades y mejor comprensión de la enfermedad, lo que lleva a una mejor calidad de vida, no solo del paciente, sino que de toda su familia y círculo de apoyo ${ }^{5}$.

Mejorar la calidad de la atención es un desafío y depende en gran medida de las perspectivas de los usuarios y las actitudes y comportamientos de los profesionales de la salud, lo que puede influir de manera beneficiosa en el control de la enfermedad ${ }^{2}$. La promoción de la autogestión efectiva requiere que los pacientes estén equipados con un repertorio de conocimientos y habilidades pertinentes a través de una educación adecuada y sistemas de apoyo ${ }^{4}$.

\section{Discusión}

La literatura reporta una gran cantidad de factores psicosociales asociados a la DMT1, especialmente en adolescentes. Diversos estudios asocian esta enfermedad con problemas psicosociales que interfieren en el control glicémico y sus relaciones familiares y sociales. Es relevante realizar intervenciones que atiendan estos aspectos, incluyendo al equipo profesionales de salud mental.

Los adolescentes con enfermedades crónicas tienen mayores conductas de riesgo, es así como los adolescentes con DMT1 presentan factores psicosociales adversos que promueven comportamientos de alto ries$\mathrm{go}^{7,30}$. Lo anterior es aún más significativo dado el bajo número de adolescentes con síntomas depresivos que reciben tratamiento psicológico ${ }^{12}$.

La educación no ha sido de gran impacto sobre el control glicémico, es por esto que se requieren intervenciones interdisciplinarias incluyendo los aspectos médicos y psicosociales de las personas y sus familias, potenciando habilidades de afrontamiento y resolución de problemas ${ }^{5,44}$. Es importante que el equipo interdisciplinario que trabaje con pacientes con DMT1 y sus familias debe mantener un contacto regular, enfatizando la participación familiar en el manejo de la diabetes ${ }^{45}$. Estudios previos han sugerido que los factores familiares tienen un mayor impacto que los tratamien- tos de insulina ${ }^{46}$, y el apoyo de pares también tendría incidencia en el autocuidado de la diabetes ${ }^{6}$.

Otro factor relevante es el sexo, evidenciándose un nivel de actividad física realizada por las niñas mucho menor que en los niños, por lo que sería un factor de riesgo para ellas. Así mismo, durante la pubertad, con los cambios hormonales, las mujeres tienen un peor manejo de la diabetes, produciendo así un incremento en las complicaciones ${ }^{30}$. Además, las mujeres presentan menores puntajes en evaluaciones de calidad de vida, problema que no tiene una explicación clara, pero podría deberse a que las adolescentes tienen más preocupaciones psicosociales y emocionales ${ }^{47}$.

En cualquier caso, los adolescentes con DMT1 deben tomar responsabilidades en el manejo de su enfermedad, pero siempre con el apoyo de su familia, para así tener una buena transición a la atención adulta ${ }^{45}$. Un ejemplo de esto son las intervenciones TEENCOPE (programa de capacitación en habilidades para hacer frente a la diabetes) y Managing Diabetes (programa de educación sobre la diabetes) ${ }^{37,48}$. Ambas intervenciones obtuvieron buenos resultados en cuanto a mejoría de puntuación de Calidad de Vida y una leve mejoría en los niveles de $\mathrm{HbAlc}^{48}$. También destaca el estudio CASCADE (Enfoque de Competencias Estructuradas de Niños y Adolescentes para la Educación sobre la Diabetes), programa educativo grupal estructurado y basado en la clínica que incorpora enfoques psicológicos con la finalidad de mejorar el control glucémico a largo plazo, la calidad de vida y el funcionamiento psicosocial en una amplia gama de niños, niñas y adolescentes diabéticos. Los resultados de este estudio demostraron que hubo una mejoría en las relaciones familiares, mejores conocimientos y comprensión, mayor confianza y mayor motivación para controlar su diabetes ${ }^{49}$.

Lo anterior, revela la necesidad de buscar otras formas para mejorar el control de la DMT $1^{8}$, una de estas podría ser incluir a profesionales en salud mental y conductual para niños y adolescentes dentro del equipo interdisciplinario, tales como psicólogos, trabajadores sociales y psiquiatras ${ }^{45}$, generando intervenciones y programas con abordaje psicosocial en nuestro país.

\section{Conflicto de intereses}

Los autores declaran no tener conflicto de intereses.

\section{Financiación}

Fundación Kimntrum. 


\section{Referencias}

1. Buschur EO, Lawrence S. Diabetes Mellitus (Type 1). In Care of Adults with Chronic Childhood Conditions. Springer International Publishing 2016;131-47.

2. Lowes L, Eddy D, Channon S, McNamara R, Robling M, Gregory JW, et al. The experience of living with type 1 diabetes and attending clinic from the perception of children, adolescents and carers: analysis of qualitative data from the DEPICTED study. J Pediatr Nurs 2015;30(1):54-62.

3. Fortin A, Rabasa-Lhoret R, Roy-Fleming A, Desjardins K, Brazeau AS, Ladouceur $\mathrm{M}$, et al. Practices, perceptions and expectations for carbohydrate counting in patients with type 1 diabetes-Results from an online survey. Diabetes Res Clin Pract 2017;126:214-21.

4. Abubakari AR, Cousins R, Thomas C, Sharma D, Naderali EK. Sociodemographic and clinical predictors of self-management among people with poorly controlled type 1 and type 2 diabetes: the role of illness perceptions and self-efficacy. J Diabetes Res 2016;6708164.

5. Anderson B. Psychosocial care for young people with diabetes. Pediatr Diabetes 2009;10(13):3-8.

6. Boogerd EA, Noordam C, Kremer JA, Prins JB, Verhaak CM. Teaming up: feasibility of an online treatment environment for adolescents with type 1 diabetes. Pediatr Diabetes 2014;15(5):394402.

7. Stahl-Pehe A, Landwehr S, Lange KS, Bächle C, Castillo K, Yossa R, et al. Impact of quality of life (QoL) on glycemic control (HbAlc) among adolescents and emerging adults with long-duration type 1 diabetes: A prospective cohortstudy. Pediatr Diabetes 2017;18(8):80816.

8. Burner ER, Menchine MD, Kubicek K, Robles M, Arora S. Perceptions of successful cues to action and opportunities to augment behavioral triggers in diabetes self-management: qualitative analysis of a mobile intervention for low-income Latinos with diabetes. J Med Internet Res 2014;16(1):e25.

9. Phillips JE, Couper JJ, Penno MA, Harrison LC. Type 1 diabetes: a disease of developmental origins. Pediatr Diabetes 2017;18(6):417-21.

10. Clapin H, Hop L, Ritchie E, Jayabalan R, Evans M, Browne-Cooper K, et al. Home-based vs inpatient education for children newly diagnosed with type 1 diabetes. Pediatr Diabetes 2017;18(7):57987.

11. Keller M, Attia R, Beltrand J, Djadi-Prat
J, Nguyen-Khoa T, Jay JP, et al. Insulin regimens, diabetes knowledge, quality of life, and $\mathrm{HbAlc}$ in children and adolescents with type 1 diabetes. Pediatr Diabetes 2017;18(5):340-7.

12. Wit $\mathrm{M}$, Winterdijk $\mathrm{P}$, Aanstoot $\mathrm{HJ}$, Anderson B, Danne T, Deeb L, et al. Assessing diabetes-related quality of life of youth with type 1 diabetes in routine clinical care: the MIND Youth Questionnaire (MY-Q). Pediatr Diabetes 2012;13(8):638-46.

13. Plener PL, Molz E, Berger G, Schober E, Mönkemöller K, Denzer C, et al. Depression, metabolic control, and antidepressant medication in young patients with type 1 diabetes. Pediatric Diabetes 2015;16(1):58-66.

14. Fry-Bowers EK, Maliski S, Lewis MA, Macabasco-O'Connell A, DiMatteo R. Health literacy and interpersonal interactions as predictors of maternal perception of ambulatory care for lowincome, Latino children. Patient Educ Couns 2013;91(2):213-20.

15. Ortiz M, Ortiz E. Adherencia al tratamiento en adolescentes diabéticos tipo 1 chilenos: una aproximación psicológica. Rev Med Chile 2005;133(3):307-13

16. Arredondo A, De Icaza E. Costos de la diabetes en América Latina: evidencias del Caso Mexicano. Value Health 2011;14(5):S85-S88.

17. Karachaliou F, Athanasakis K, Tsentidis C, Soldatou A, Simatos G, Kyriopoulos $\mathrm{J}$, et al. A cohort of children with type 1 diabetes in Greece: predictors of direct costs of care. Pediatr Diabetes 2017;18(5):405-12.

18. Zimmet P, Alberti KGM, Kaufman F, Tajima N, Silink M, Arslanian S, et al. The metabolic syndrome in children and adolescents-an IDF consensus report. Pediatr Diabetes 2007;8(5):299306.

19. Guía clínica AUGE Diabetes Mellitus tipo 1. Series Guías Clínicas MINSAL, 2013. [Disponible en http://www. bibliotecaminsal.cl/wp/wp-content/ uploads/2016/04/Diabetes-Mellitustipo-1.pdf].

20. Listado de prestaciones específicas. Anexo Decreto Régimen de Garantías Explicitas en Salud, Ministerio de Salud, 2016 [Disponible en http://web.minsal.cl/ portal/url/item/d6924d336146d5e6e04001 0164015e8f.pdf]

21. Protocolo 2016. Tratamiento basado en la administración de insulina a través de infusores subcutáneos continuos de insulina (bombas de insulina) para personas con diagnóstico de diabetes tipo 1 inestable severa. Para el Otorgamiento de las Prestaciones que cuentan con el Sistema de Protección Financiera para Diagnósticos y Tratamiento de Alto Costo. Ley 20.850. [Disponible en http://web.minsal.cl/wp-content/ uploads/2015/08/4_Protocolo-Bombas-1. pdf].

22. Rosolowsky E, Yaskina M, Couch R. Pediatric Type 1 Diabetes: Patients' and Caregivers' Perceptions of Glycemic Control. Can J Diabetes 2017: S14992671(17)30281-2.

23. Lindström C, Åman J, Norberg AL. Increased prevalence of burnout symptoms in parents of chronically ill children. Acta paediatrica 2010;99(3):42732 .

24. Lindström C, Åman J, Norberg AL. "Parental burnout in relation to sociodemographic, psychosocial and personality factors as well as disease duration and glycaemic control in children with Type 1 diabetes mellitus". Acta Paediatr 2011;100(7):1011-7.

25. Noser AE, Huffhines L, Clements MA, Patton SR. Diabetes conflict outstrips the positive impact of self-efficacy on youth adherence and glycemic control in type 1 diabetes. Pediatr Diabetes 2017;18(7):6148 .

26. Melzer SM, Richards GE, Covington ML. Reimbursement and costs of pediatric ambulatory diabetes care by using the resource-based relative value scale: is multidisciplinary care financially viable? Pediatr Diabetes 2004;5(3):133-42.

27. Jackson C, Richer J, Edge JA. Sibling psychological adjustment to type 1 diabetes mellitus. Pediatric Diabetes 2008;9(Part I):308-11.

28. Särnblad S, Berg L, Detlofsson I, Jönsson $\AA$, Forsander G. Diabetes management in Swedish schools: a national survey of attitudes of parents, children, and diabetes teams. Pediatr Diabetes 2014;15(8):550-6.

29. Driscoll KA, Volkening LK, Haro H, Ocean G, Wang Y, Jackson CC, et al. Are children with type 1 diabetes safe at school? Examining parent perceptions. Pediatr Diabetes 2015;6(8):613-20.

30. Forsander G, Bøgelund M, Haas J, Samuelsson U. Adolescent life with diabetes-Gender matters for level of distress. Experiences from the national TODS study. Pediatr Diabetes 2017;18(7):651-9.

31. Baucom KJ, Queen TL, Wiebe DJ, Turner SL, Wolfe KL, Godbey EI, et al. Depressive symptoms, daily stress, and adherence in late adolescents with type 1 diabetes. Health Psychol 2015;34(5):52230.

32. Cooper MN, Lin A, Alvares GA, de Klerk $\mathrm{NH}$, Jones TW, Davis EA. Psychiatric disorders during early adulthood in those with childhood onset type 1 diabetes: Rates and clinical risk factors from 
population-based follow-up. Pediatr Diabetes 2017;18(7):599-606.

33. Ortiz MS, Myers HF. Control metabólico en pacientes diabéticos tipo 1 chilenos: rol del estrés psicológico. Rev Med Chile 2014;142(4):451-7.

34. Hermann JM, Meusers M, Bachran R, Kuhnle-Krahl U, Jorch N, Hofer SE, Holl RW. Self-reported regular alcohol consumption in adolescents and emerging adults with type 1 diabetes: A neglected risk factor for diabetic ketoacidosis? Multicenter analysis of 29630 patients from the DPV registry. Pediatr Diabetes 2017;18(8):817-23.

35. Agarwal S, Garvey KC, Raymond JK, Schutta MH. Perspectives on care for young adults with type 1 diabetes transitioning from pediatric to adult health systems: a national survey of pediatric endocrinologists. Pediatr Diabetes 2017;18(7):524-31.

36. McGrady ME, Hood KK. Depressive symptoms in adolescents with type 1 diabetes: associations with longitudinal outcomes. Diabetes Res Clin Pract 2010;88(3):e35-e37.

37. Cadario F, Prodam F, Bellone S, Trada M, Binotti M, Trada M, et al. Transition process of patients with type 1 diabetes (T1DM) from paediatric to the adult health care service: a hospital-based approach. Clin Endocrinol (Oxf) 2009;71:346-50.

38. Zubarew T, Correa L, Bedregal P, Besoain C, Reinoso A, Velarde M, et al. Transición de adolescentes portadores de enfermedades crónicas desde servicios pediátricos a servicios de adultos: Recomendaciones de la Rama de Adolescencia de la Sociedad Chilena de Pediatría. Rev Chil Pediatr 2017;88(4):553-60.

39. American Diabetes Association. Standards of medical care in diabetes-2016 abridged for primary care providers. Clinical diabetes: a publication of the American Diabetes Association 2016;34(1):3. (Disponible en http://professional. diabetes.org/standards)

40. Chamorro MM, Martínez IL, Tomás CL. Perfil psicosocial de niños y adolescentes con diabetes mellitus. Boletín de Pediatría 2002;42:114-9.

41. Ledón L. Impacto psicosocial de la diabetes mellitus, experiencias, significados y respuestas a la enfermedad. Rev Cubana Endocrinol 2012;23(1):76-97.

42. Eilander MM, de Wit M, Rotteveel J, Aanstoot HJ, Bakker-van Waarde WM, Houdijk E, et al. "Diabetes IN development (DINO): the biopsychosocial, family functioning and parental well-being of youth with type 1 diabetes: a longitudinal cohort study design". BMC pediatrics 2015;15(1):82.

43. Fava G, Nicoletta S. "From the Lesson of George Engel to Current Knowledge: The Biopsychosocial Model 40 Years Later”. Psychotherapy and psychosomatics 2017;86(5):257-9.

44. Floyd BD, Block JM, Buckingham BB, Ly $\mathrm{T}$, Foster N, Wright R, et al. Stabilization of glycemic control and improved quality of life using a shared medical appointment model in adolescents with type 1 diabetes in suboptimal control. Pediatr Diabetes 2017;18(3):20412.

45. Delamater AM, de Wit M, McDarby V, Malik J, Acerini CL. Psychological care of children and adolescents with type 1 diabetes. Pediatr Diabetes 2014;15(20):232-44

46. Martin D, Elie C, Dossier C, Godot C, Gagnayre R, Choleau C, et al. Diabetes knowledge in adolescents with type 1 diabetes and their parents and glycemic control. Pediatr Diabetes 2017;18(7):55965.

47. Naughton MJ, Joyce P, Morgan TM, Seid M, Lawrence JM, Klingensmith GJ, et al. Longitudinal associations between sex, diabetes self-care, and health-related quality of life among youth with type 1 or type 2 diabetes mellitus. J Pediatr 2014; 164(6):1376-83.

48. Grey M, Whittemore R, Jeon S, Murphy K, Faulkner MS, Delamater A, Teen Cope Study Group. Internet psycho-education programs improve outcomes in youth with type 1 diabetes. Diabetes Care 2013;36(9):2475-82.

49. Christie D, Thompson R, Sawtell M, Allen E, Cairns J, Smith F, et al. Structured, intensive education maximising engagement, motivation and long-term change for children and young people with diabetes: a cluster randomised controlled trial with integral process and economic evaluation-the CASCADE study. Health Technol Assess. 2014;18(20):1. 